\title{
Traumatic Brachiocephalic Artery Pseudoaneurysm
}

\section{Sally Shepherd" and Jessica Wong}

Department of Trauma and General Surgery, The Royal Melbourne Hospital, Victoria, Australia

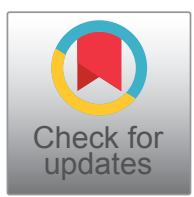

*Corresponding author: Sally Shepherd, Department of Trauma and General Surgery, The Royal Melbourne Hospital, Victoria, Australia

A 19-year-old female presented to a tertiary Trauma Centre post intentional $100 \mathrm{~km} / \mathrm{hr}$ motor vehicle crash into a tree. She was wearing a seatbelt in the driver's seat, airbags were deployed and required assistance extricating after entrapment for 45 minutes. She was normotensive but tachycardic with a shortened and rotated right lower limb, receiving $500 \mathrm{mls}$ crystalloid and splinting to her right lower limb.

On arrival in ED her airway was patent and cervical spine secured in a rigid collar. She was saturating $98 \%$ on room air with an obvious seatbelt sign across the chest. She was tachycardic at 125 beats per minute, with cool peripheries, and weak pulses in bilateral lower limbs. She was GCS 15 with $3 \mathrm{~mm}$ reactive pupils bilaterally and hypothermic at 36 degrees.

A CXR showed mild upper mediastinal widening and a pelvic XR with no abnormalities. 2 large bore intravenous cannulas were inserted and she was resuscitated with further $1 \mathrm{~L}$ crystalloid.

She was stabilized for a CT trauma series (CT brain, cervical spine, CT arterial phase of chest, abdomen and pelvis, CT portal venous phase of abdomen and pelvis, and gated CT chest) which revealed a grade 3a aortic arch transection with brachiocephalic pseudoaneury$\mathrm{sm}$, a grade 2 AAST splenic and grade 3 AAST liver laceration, a zone 1 retroperitoneal haematoma, small bilateral pneumothoraces and a type 2 right open femoral fracture.

Her right lower limb open wound was washed and covered with betadine gauze. It was then placed in 3 kg skin traction with a femoral nerve block performed for analgesia. She did not require intercostal catheter insertion as saturations remained greater than $98 \%$ wi- thout supplemental oxygen and no signs of respiratory distress.

Her past medical history included depression only with previous deliberate self harm but nil suicide attempts and she was not on any regular medications.

Endovascular repair of the brachiocephalic artery was attempted. A stent was placed across the brachiocephalic artery which then migrated to the infrarenal abdominal aorta. A second brachiocephalic artery stent was then placed but had persistent leak at the base. The case was abandoned and referral made to Cardiothoracic Surgery.

She returned to Intensive Care where a right internal jugular pulmonary artery sheath was inserted and her systolic blood pressure was maintained less than 120 $\mathrm{mmHg}$ with labetalol and glyceryl trinitrate.

She returned to theatre 5 hours later for a median sternotomy, aortic arch repair with a $8 \mathrm{~mm}$ graft, extraction of brachiocephalic artery stent and excision of innominate artery pseudoaneurysm. Cardiopulmonary bypass was performed for 2 hours with 49 minutes of cross clamp time. Orthopaedic surgery followed by washing and closing her wounds and placing the right lower limb in external fixation which was definitively managed one day later.

She returned to ICU where she was extubated day 2 post-operation with no neurological consequences. Pacing wires were removed day 3 post-operation and transferred to the ward from day 4 post-operation. Her recovery was complicated by bilateral pleural effusions that responded to frusemide. She was discharged home 10 days post-operative repair.

\footnotetext{
Citation: Shepherd S, Wong J (2021) Traumatic Brachiocephalic Artery Pseudoaneurysm. Trauma Cases Rev 7:089. doi.org/10.23937/2469-5777/1510089 in any medium, provided the original author and source are credited.
} 


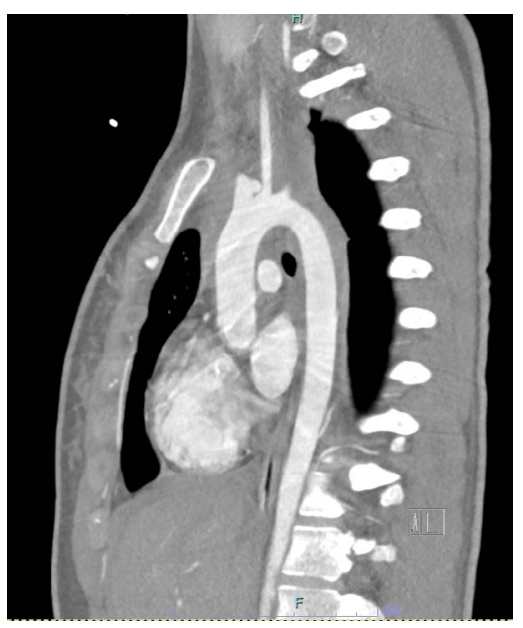

A.
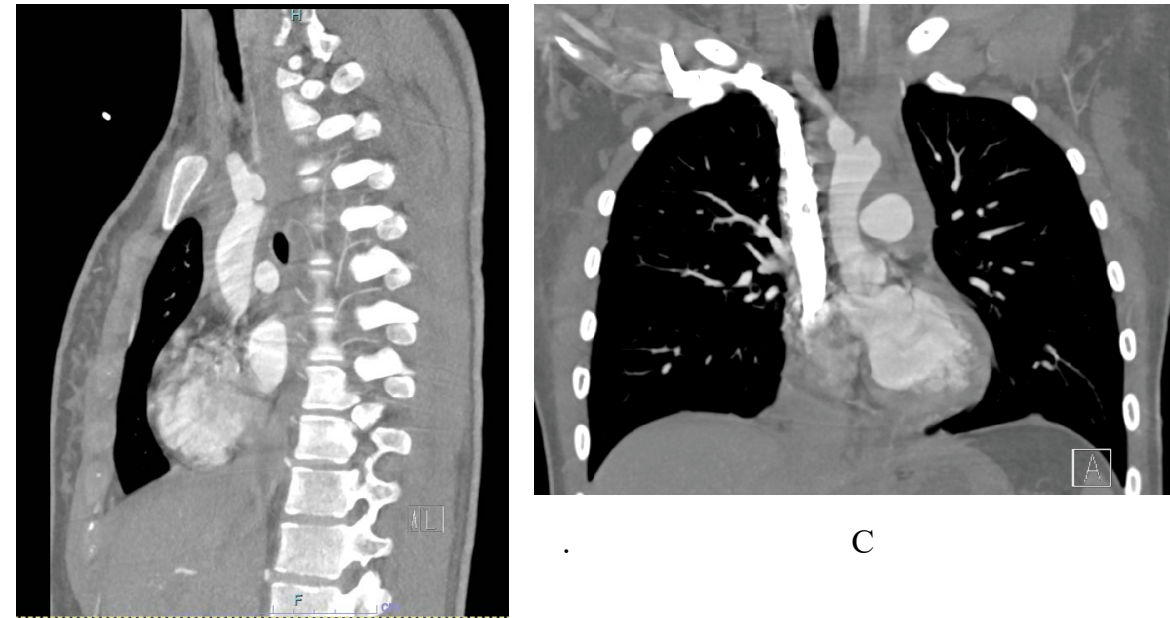

$\mathrm{C}$

Figure 1: CT arterial phase of the chest. (A-B) Sagittal slices; (C) Coronal slice revealing a pseudoaneurysm arising from the posterior aspect of the proximal right brachiocephalic artery.

\section{Discussion}

Injury to the brachiocephalic artery is a rare consequence of blunt chest trauma but must be recognised as it is potentially life threatening. Chest X-Ray is a useful adjunct for diagnosis, but CT arterial phase and gated CT chest are most useful in delineating the exact location of injury and guiding treatment [1].

Traditionally, brachiocephalic artery injuries were approached with a median sternotomy, which itself can contribute to significant morbidity from long operative times and post-operative complications. However, a move to endovascular treatment with covered stents has seen a reduction in morbidity and mortality. Endovascular treatment for this condition is limited but is a good option as it avoids a median sternotomy cardiopulmonary bypass and the potential complications of cardiopulmonary bypass [2-4].

Patients with traumatic injuries to the brachiocephalic artery as a result of blunt chest injury must be promptly treated to avoid significant morbidity or mortality, with a multidisciplinary approach. In the event that Endovascular treatment is unsuccessful, a prompt intervention by a Cardiothoracic team for median sternotomy must be available.

\section{Conclusion}

This case highlights that brachiocephalic artery injury is a rare, but potentially lethal injury as a result of blunt chest trauma. Safe management requires a combined Vascular and Cardiothoracic team approach as stenting alone may be insufficient.

\section{References}

1. Fisher R, Chasen M, Neela L (1994) Diagnosis of injuries of the aorta and brachiocephalic arteries caused by blunt chest trauma: CT vs aortography. AJR Am J Roentgenol 162: 1047-1052.

2. Shalhub S, Starnes BW, Hatsukami TS, Karmy-Jones R, Tran NT (2011) Repair of blunt thoracic outlet arterial injuries: An evolution from open to endovascular approach. $\mathrm{J}$ Trauma 71: E114-E121.

3. Rousseau H, Elaassar O, Marcheix B, Cron C, Valérie C, et al. (2011) The Role of stent-grafts in the management of aortic trauma. Cardiovasc Intervent Radiol 35: 2-14.

4. Kolbeck KJ, Kaufman JA (2011) Endovascular stent grafts in urgent blunt and penetrating thoracic aortic trauma. Semin Intervent Radiol 28: 98-106.

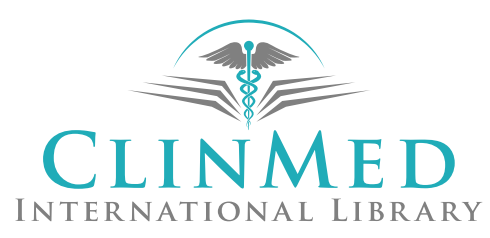

